\title{
Archéopages
}

Archéopages

Archéologie et société

40 | 04-07/2014

Villages

\section{L'archéologie dans le village. Quelques exemples récents d'opérations préventives en Val de Loire}

Stéphane Joly, Jean-Philippe Chimier et Laurent Fournier

\section{(2) OpenEdition}

\section{Journals}

Édition électronique

URL : https://journals.openedition.org/archeopages/615

DOI : 10.4000/archeopages.615

ISSN : 2269-9872

\section{Éditeur}

INRAP - Institut national de recherches archéologiques préventives

\section{Édition imprimée}

Date de publication : 1 juin 2015

Pagination : 78-83

ISSN : $1622-8545$

\section{Référence électronique}

Stéphane Joly, Jean-Philippe Chimier et Laurent Fournier, «L'archéologie dans le village. Quelques exemples récents d'opérations préventives en Val de Loire ", Archéopages [En ligne], 40 | 04-07/2014, mis en ligne le 01 juillet 2016, consulté le 25 janvier 2022. URL : http://journals.openedition.org/ archeopages/615; DOI : https://doi.org/10.4000/archeopages.615 


\section{L'archéologie dans le village Quelques exemples récents d'opérations préventives en Val de Loire}

Stéphane Joly Inrap, UMR 7324 “Cites, territioires, environnement t t societen"

Jean-Philippe Chimier Inrap, UMR 7324 «Citess, territiores, environnementet societte"

Laurent Fournier Inrap

Les interventions d'archéologie préventive au sein des villages actuels se sont multipliées récemment avec l'essor de la discipline. Ces diagnostics, qui donnent rarement lieu à des fouilles, apportent malgré tout une documentation archéologique exploitable. Les exemples sont nombreux dans le Val de Loire: il s'agit pour beaucoup d'interventions dans ou en périphérie des pôles ecclésiaux, portant plus rarement sur l'habitat ou l'artisanat comme les quatre études de cas présentées ici [ill. 1]. Elles sont représentatives de cette documentation nouvelle, ainsi que de la diversité des résultats et des méthodes mises en œuvre.

\section{D'une agglomération antique au village : l'exemple de Bonnée (Loiret)}

Le bourg actuel de Bonnée est situé dans le Val d'Orléans. La présence d'une agglomération antique (Cribellier, 1999) a motivé la réalisation de sept diagnostics depuis 2001, dans ou en périphérie immédiate du village (Joly et al., 2014). Ces interventions sont toutes positives à divers degrés. Elles couvrent au total 8,4 ha et 531 vestiges ont été identifiés [ill. 2]. En l'absence de décapage extensif et de fouille, les données archéologiques sont fragmentaires et leurs interprétations parfois délicates. À l'échelle du village, elles permettent d'entrevoir la parfaite continuité de l'occupation et l'évolution d'une agglomération du $\mathrm{I}^{\text {er }}$ siècle jusqu'à nos jours.

L'agglomération antique, qui possède une parure monumentale assurée mais mal caractérisée, s'étendrait sur au moins une vingtaine d'hectares, sans doute le long d'un axe de circulation. À partir du Bas-Empire, on observe deux phénomènes concomitants: une nette décrue apparente de l'occupation au sein de l'agglomération initiale et l'apparition d'habitats sur des terrains autrefois périphériques. Ainsi, en bordure sud-est s'installe, sur 1,5 ha, un habitat occupé de la fin du $v^{\mathrm{e}}$ au début du viII ${ }^{\mathrm{e}}$ siècle (Joly et al., 2008). Avec ses constructions sur poteaux, plusieurs fours et foyers, trois sépultures et un possible fond de cabane, il ne se différencie pas des établissements ruraux habituels. Sa taille est déjà notable, mais il s'étend apparemment vers l'ouest, où est situé le théâtre antique. Les ruines de celui-ci, encore visibles au XIX ${ }^{\mathrm{e}}$ siècle (Jollois, 1836, p. 56-61), laissent supposer qu'il a sans doute continué à jouer un certain rôle au Moyen Âge, ne serait-ce que dans la topographie. Dans ce secteur se trouvent aussi l'église et le carrefour des principaux chemins attestés ultérieurement. À partir du VIII siècle, les données archéologiques se tarissent, seuls deux fours domestiques datant peut-être de la fin du Moyen Âge ayant été identifiés plus au nord-est (De Filippo, 2012).

Les sources écrites démontrent l'existence d'un habitat pourvu d'un lieu de culte à partir du IX ${ }^{\mathrm{e}}$ siècle, voire dès le VII ${ }^{\mathrm{e}}$ siècle (Jesset, 2012, p. 120). Une chronique du $\mathrm{IX}^{\mathrm{e}}$ siècle relatant des miracles survenus vers 660 mentionne le praediolum Bonodium où une basilica est consacrée à saint Benoît (HTSB, 1858, p. 9-10). La continuité attestée par le croisement des sources n'exclut pas une certaine mobilité de l'occupation. Les occupations archéologiques du centre-bourg sont mal connues car inexplorées en raison du bâti actuel. L'agglomération antique s'y étend certainement. L'habitat médiéval et moderne peut aussi s'y être développé, ce qui préfigure l'état illustré sur le cadastre de 1837. 
1. Localisation des sites présentés.

2. Cartes de synthèse des occupations de Bonnée. La présence d'une

agglomération antique

à Bonnée a motivé la

réalisation de sept

diagnostics depuis 2001,

dans ou en périphérie

immédiate du village.

Ces interventions, qui

couvrent au total 8,4 ha,

ont permis d'identifier

531 vestiges.

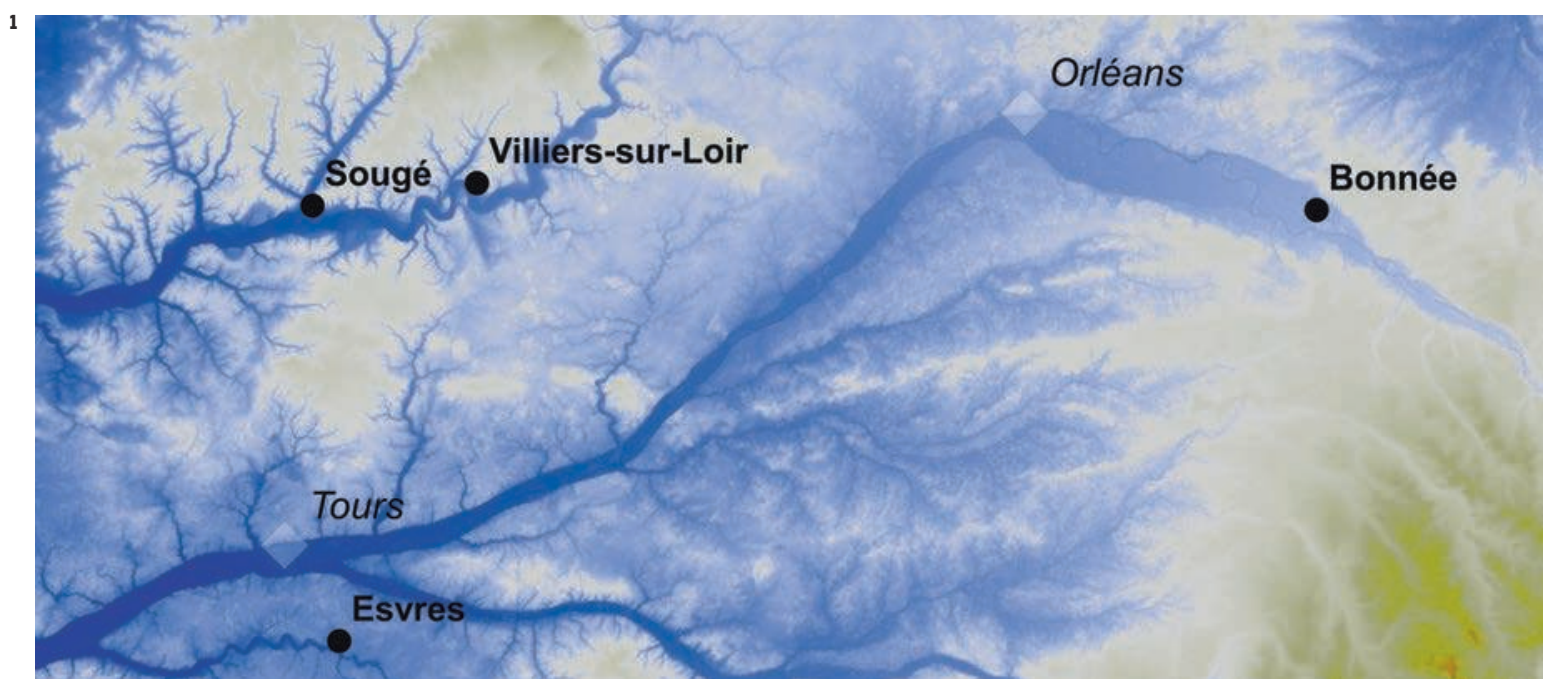

$\stackrel{N}{\Lambda}$

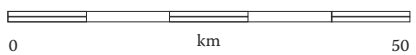

2
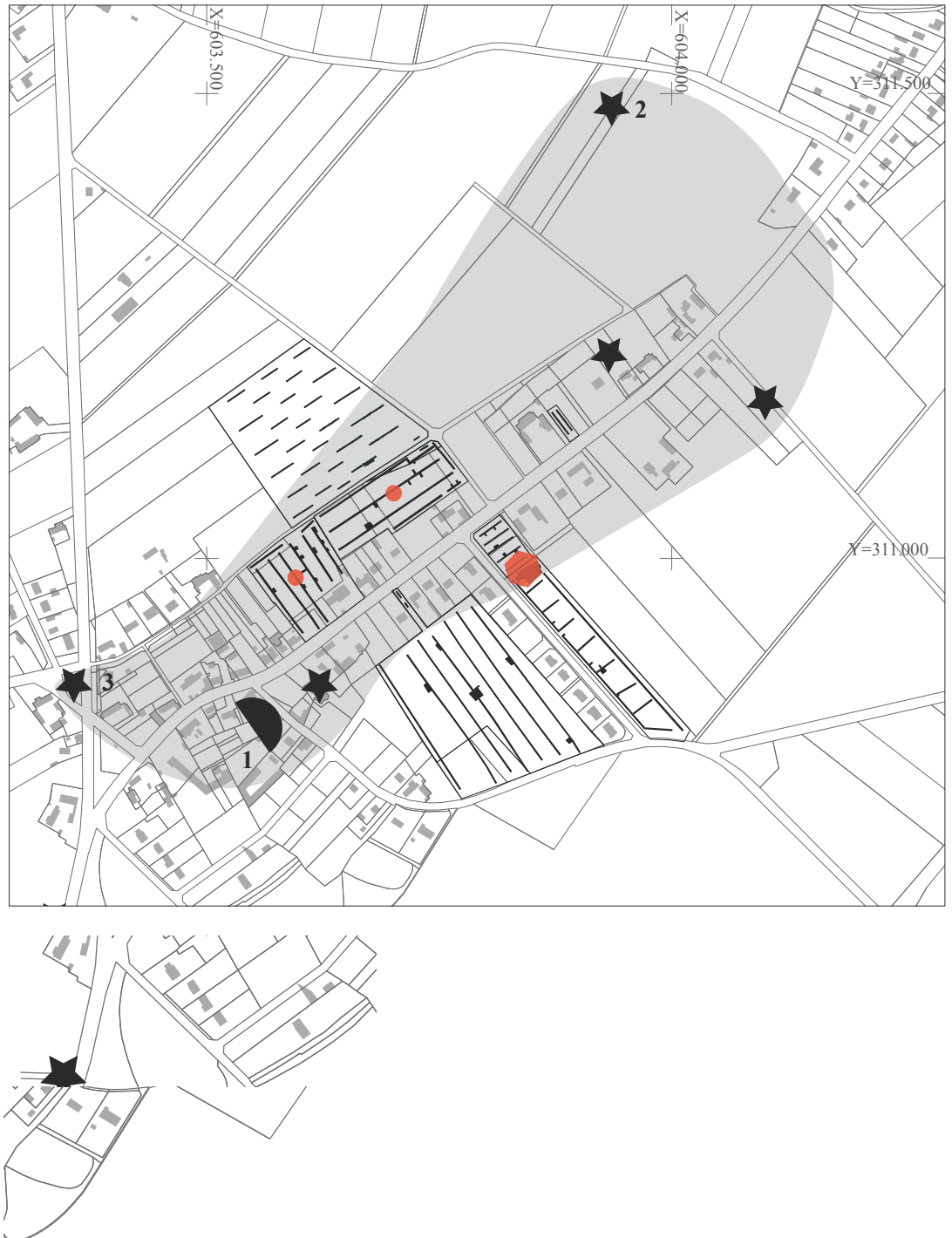

$\stackrel{\mathrm{N}}{\rightleftharpoons} \stackrel{\mathrm{m}}{\rightleftharpoons}$
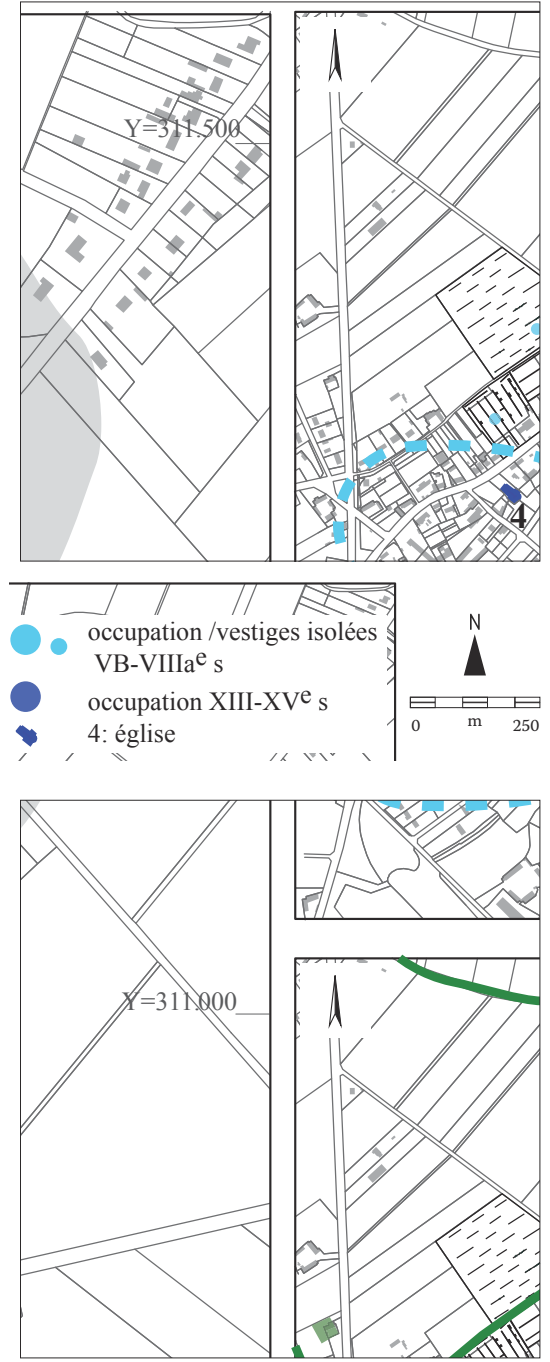

parcelles bâties en 1837

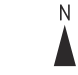

(2)

章 
3. Esvres antique. 4. Esvres, cadastre de 1823 et courbes de niveau Le village se compose de plusieurs espaces: le château, en contrebas duquel se situe l'église, est aménagé sur une plate-forme. L'habitat s'organise dans l'anomalie et le long des voies.

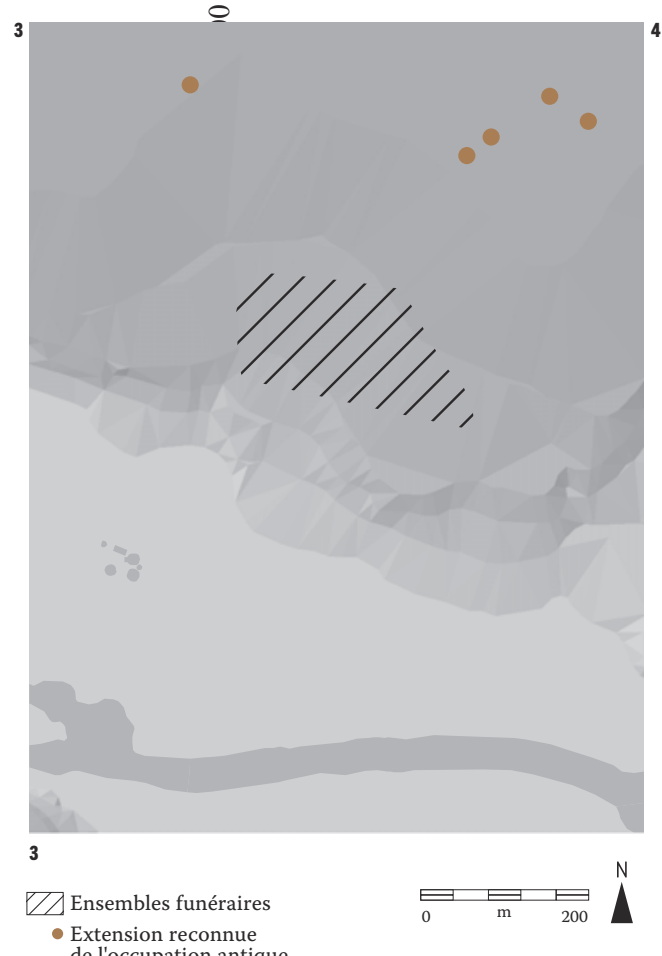

de l'occupation antique

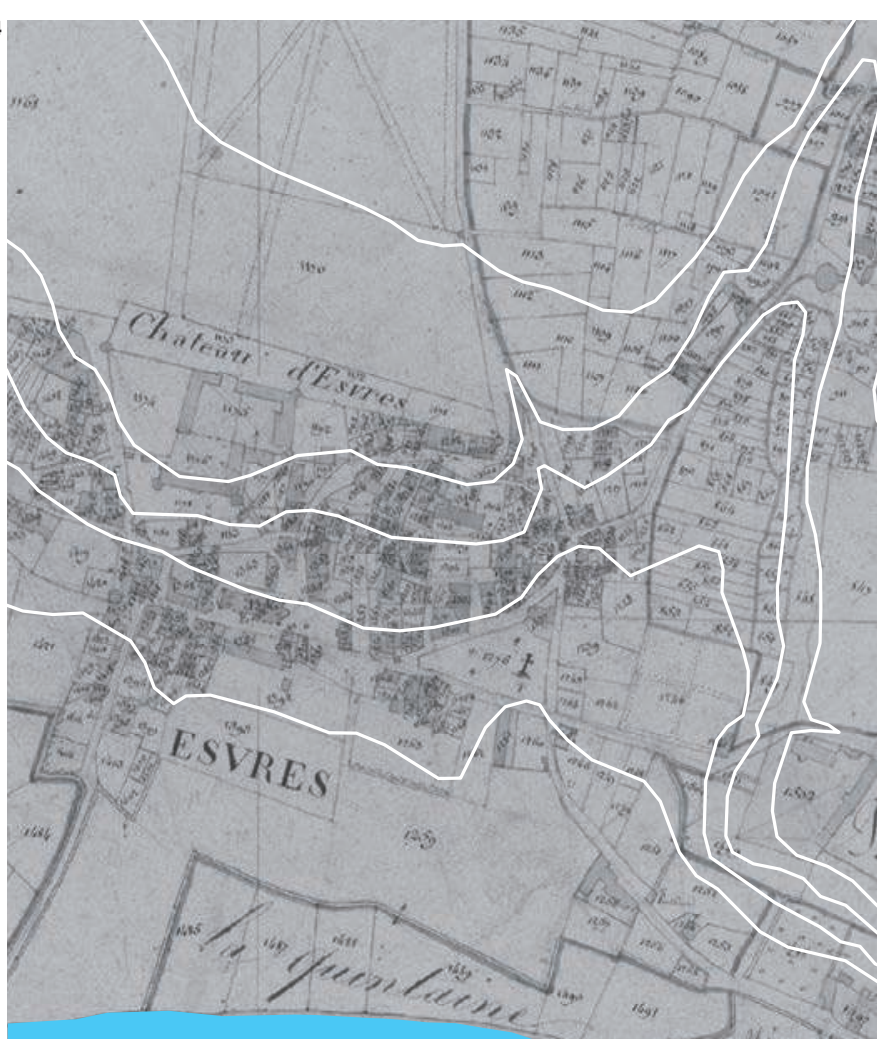

$\rightleftharpoons \rightleftharpoons$
Le programme de recherche Evena: l'étude du village d'Esvres (Indre-et-Loire) dans la longue durée

Esvres est situé dans la vallée de l'Indre, sur le versant du coteau nord. Depuis 2011, un programme de recherche a pour objectif l'évaluation archéologique du village et de son environnement rural. Les informations proviennent de sources différentes : l'étude de la documentation archivistique et du patrimoine bâti, les données archéologiques anciennes, les opérations d'archéologie préventive et une opération programmée associant prospections au sol et sondages manuels. Le bâti actuel occultant les occupations antérieures, les sources archéologiques s'y rapportant proviennent uniquement de sondages, programmés ou de diagnostics (Chimier, Fouillet, 2012 ; Chimier, Fouillet, 2013).

Les plus anciennes occupations correspondent à un habitat daté du i ${ }^{\mathrm{er}}$ siècle avant notre ère et du Haut-Empire, localisé sur le rebord du plateau [ill. 3]. En l'absence de décapage extensif, l'hypothèse d'une agglomération se fonde sur deux éléments : la présence d'une vaste aire funéraire et la mention d'un vicus au vi ${ }^{\mathrm{e}}$ siècle (Chimier et al., 2014). Les nécropoles comprennent plus d'une centaine de tombes et sont utilisées du $\mathrm{II}^{\mathrm{e}}$ siècle avant notre ère $\mathrm{au} \mathrm{II}^{\mathrm{e}}$ siècle de notre ère. La diversité des sépultures suggère qu'elles relèvent d'une agglomération plutôt que d'un établissement rural: une part de la population inhumée correspond à une classe sociale privilégiée, illustrée par la présence de sépultures à arme et amphore (Chimier, Riquier, 2009). Le site apparaît dans les textes au vi ${ }^{\mathrm{e}}$ siècle: Evena est un des 31 vici de Touraine mentionnés par Grégoire de Tours (Zadora-Rio, 2008, p. 77-79). L'évêque relate qu'une église y a été fondée dans la seconde moitié $\mathrm{du} \mathrm{v}^{\mathrm{e}}$ siècle. Aucune donnée archéologique n'est toutefois disponible pour cette période et la nature du vicus, notamment la morphologie de l'habitat, n'est pas connue. Au viI ${ }^{\mathrm{e}}$ siècle, le vicus est un lieu d'émission monétaire.

Esvres est ensuite chef-lieu de viguerie du viII ${ }^{\mathrm{e}}$ au $\mathrm{X}^{\mathrm{e}}$ siècle. Les données archéologiques sont là aussi lacunaires (ibid., p. 80-84, 90-94; Zadora-Rio, 2010). Une nécropole à sarcophages est attestée dans le secteur de l'église médiévale et se développe en contrebas du coteau jusqu'à son transfert au $\mathrm{XIX}^{\mathrm{e}}$ siècle (Chimier, 2012). L'emplacement de l'église d'origine n'est pas connu. L'édifice actuel, dont le premier état est antérieur au XIII ${ }^{\mathrm{e}}$ siècle, succède à une construction non datée (Lauliac, 2013).

Le cadastre de 1823 montre que le village se compose de plusieurs espaces [ill. 4]. Le château n'est mentionné par les textes qu'à partir du $\mathrm{XIII}^{\mathrm{e}}$ siècle. Il est aménagé sur une plate-forme en rebord du plateau et surplombe la vallée. Une anomalie parcellaire ovale marque le versant 
plus à l'est. L'église est bâtie en limite du lit majeur de l'Indre, en contrebas du château. L'habitat s'organise dans l'anomalie et le long des voies. Les sondages réalisés dans la partie ancienne du bourg n'ont pas encore livré d'informations complémentaires quant à sa nature (Chimier et al., 2014).

\section{Le bourg de Sougé (Loir-et-Cher) :} des occupations dès le premier haut Moyen Âge

Sougé est situé à la confluence des vallées du Loir et de la Braye. Trois interventions d'archéologie préventives se sont déroulées depuis 2006 au sud du bourg actuel, à une centaine de mètres de l'église, sur une emprise totalisant moins de 1,4 ha [ill. 5]. Un premier diagnostic (Thierry, 2006) suivi d'une fouille (Carlier, Ladureau, 2008) ont mis en évidence une occupation attribuable aux $\mathrm{VI}^{\mathrm{e}}$ et $\mathrm{VII}^{\mathrm{e}}$ siècles. Au sein d'un enclos fossoyé, plusieurs constructions sur poteaux ainsi que des fosses, des foyers et des déchets de forge témoignent d'une zone mixte d'habitat et d'artisanat. Le mobilier céramique, par son nombre et sa qualité, se démarque de celui habituellement rencontré. Un second diagnostic sur des terrains mitoyens suggère l'extension diffuse de cette occupation à l'est (Fournier, 2012). Au cours de cette intervention, une occupation

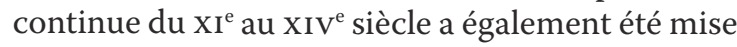
en évidence. Elle se caractérise par la présence de plusieurs bâtiments en pierre. À compter du XIII ${ }^{\mathrm{e}}$ siècle, cet habitat se densifie, avec de grands bâtiments et des constructions annexes dont un four à pain. Cette phase d'occupation a aussi été reconnue lors des premières interventions. Plusieurs cuves ou bassins, dont certains maçonnés, y témoignent d'un artisanat du cuir du XIV ${ }^{\mathrm{e}}$ au XVII ${ }^{\mathrm{e}}$ siècle.

Ces vestiges illustrent l'évolution d'un quartier dévolu à l'habitat et à l'artisanat du vi ${ }^{\mathrm{e}}$ siècle à la période moderne. Un hiatus apparent marque la fin du haut Moyen Âge. Le vocable de l'église paroissiale (saint Quentin) suggère une fondation aux VIII ${ }^{\mathrm{e}}$-XI ${ }^{\mathrm{e}}$ siècles (Barthélémy, 1993, p. 141), le presbytère daterait stylistiquement du XII ${ }^{\mathrm{e}}$ siècle et les textes confirment l'existence de l'église et de la paroisse à partir du XIII ${ }^{\mathrm{e}}$ siècle (De SaintVenant, 1917, p. 397-403). Au Moyen Âge central, la position excentrée des terrains diagnostiqués est établie, la localisation des tanneries s'explique par la relégation d'activités polluantes hors de l'habitat.

\section{Les prémices du village médiéval à Villiers-sur-Loir (Loir-et-Cher) aux VIII ${ }^{\mathrm{e}}-\mathrm{IX}^{\mathrm{e}}$ siècles?}

Le village actuel se développe au pied du coteau. Dans le bourg, seules des découvertes anciennes et fortuites d'ossements et de sépultures non datées autour de l'église sont connues (Mésange, 1995, p. 101-105). L'unique diagnostic réalisé, d'une emprise de $2511 \mathrm{~m}^{2}$, a permis la découverte de près d'une centaine de vestiges (Joly, Bouillon, 2014). Leur densité et la courte période concernée (du $\mathrm{VIII}^{\mathrm{e}}$ au $\mathrm{IX}^{\mathrm{e}}$ siècle, voire éventuellement $\mathrm{au} \mathrm{X}^{\mathrm{e}}$ siècle) confirment une intensité d'occupation assez remarquable [ill. 6].

Quelques tessons résiduels suggèrent une fréquentation antérieure, dès l'Antiquité tardive ou le début du haut Moyen Âge, confirmant indirectement les découvertes anciennes des sépultures supposées «mérovingiennes » au «Clos Amery» à 500 m environ, plus à l'ouest. Les éléments structurant l'occupation de la fin du haut Moyen Âge n'ont pas été clairement identifiés. Les diverses concentrations de trous de poteau attestent plusieurs constructions accompagnées d'une vingtaine de fosses et de silos. Une structure de combustion avec de nombreux déchets métallurgiques indique une probable activité de réduction. Ces vestiges semblent correspondre à plusieurs habitats domestiques contemporains et voisins associés à un artisanat. Le mobilier va aussi dans le sens d'une occupation domestique à caractère plutôt familial. Quelques indices de qualité de vie (nourriture carnée, verre, parois enduites) sont perceptibles. La position particulière de cet habitat aggloméré n'est pas sans évoquer un quartier du village en devenir. Pourtant, la fin apparente de l'occupation au $\mathrm{x}^{\mathrm{e}}$ siècle ne permet pas de le lier directement aux biens d'une certaine importance, tenus en main-ferme (manufirma), cités par les textes ${ }^{\mathbf{1}}$ un siècle plus tard environ, au milieu du XI ${ }^{\mathrm{e}}$ siècle (Barthélemy, 1993, p. 44-46, 388-389, 456). L'église, elle-même, est attestée peu après : la dédicace d'Hilaire évoque une datation du VIII ${ }^{\mathrm{e}}$ au XI ${ }^{\mathrm{e}}$ siècle (Barthélemy, 1993, p. 141), le bâtiment daterait stylistiquement du XII ${ }^{\mathrm{e}}$ siècle et un officiant est mentionné vers 1190 (De SaintVenant, 1917, p. 292-293).

\section{De l'apport de l'archéologie préventive pour l'étude de la diversité des trajectoires villageoises \\ Ces études de cas illustrent différentes} formations et trajectoires de villages actuels. Aucun exemple des nombreuses interventions récentes d'archéologie préventive au sein des pôles ecclésiaux n'a volontairement été présenté ${ }^{2}$. Dans tous les cas, si l'archéologie dans le village est une pratique parfois ingrate et toujours complexe, tant sur le plan administratif et technique que scientifique, ses apports ne sont plus à démontrer (Carré et al., 2013; Mahé-Hourlier, Poignant, 2013).

Les données proviennent pour l'essentiel de diagnostics d'archéologie préventive et sont peutêtre à ce titre déconsidérées ou plus simplement ignorées. Cette documentation récente et foisonnante reste par ailleurs discrète et souvent inaccessible, car non publiée. Elle est peu intégrée au sein des travaux de synthèse à l'échelle du département ou de la région, certainement en raison de sa production récente. C'est sans doute le cas du travail d'Elisabeth Zadora-Rio sur les 

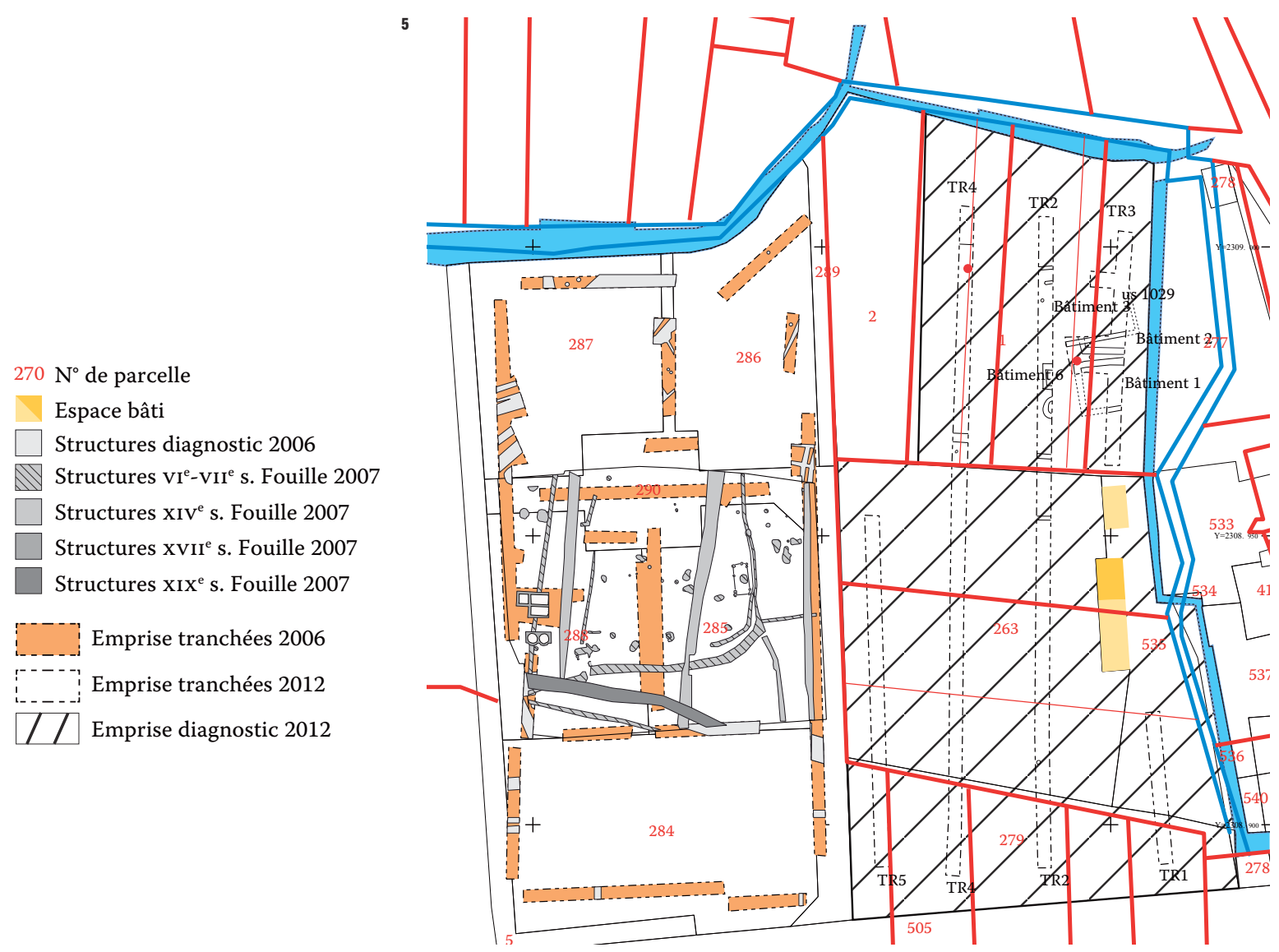

5. Localisation des diagnostics et fouilles de Sougé sur le plan parcellaire actuel. Depuis 2006, trois interventions d'archéologie préventives se sont déroulées au sud du bourg actuel, sur une emprise totalisant moins de 1,4 ha. Les vestiges mis au jour illustrent l'évolution d'un quartie dévolu à l'habitat et à l'artisanat du vi' siècle à la période moderne. 6. Localisation du diagnostic et des vestiges archéologiques de Villiers-sur-Loir sur le plan parcellaire actuel. D'une emprise de $2511 \mathrm{~m}^{2}$, I'unique diagnostic réalisé a permis la découverte de près d'une centaine de vestiges. Leur densité et la courte période concernée confirment une intensité d'occupation assez remarquable.
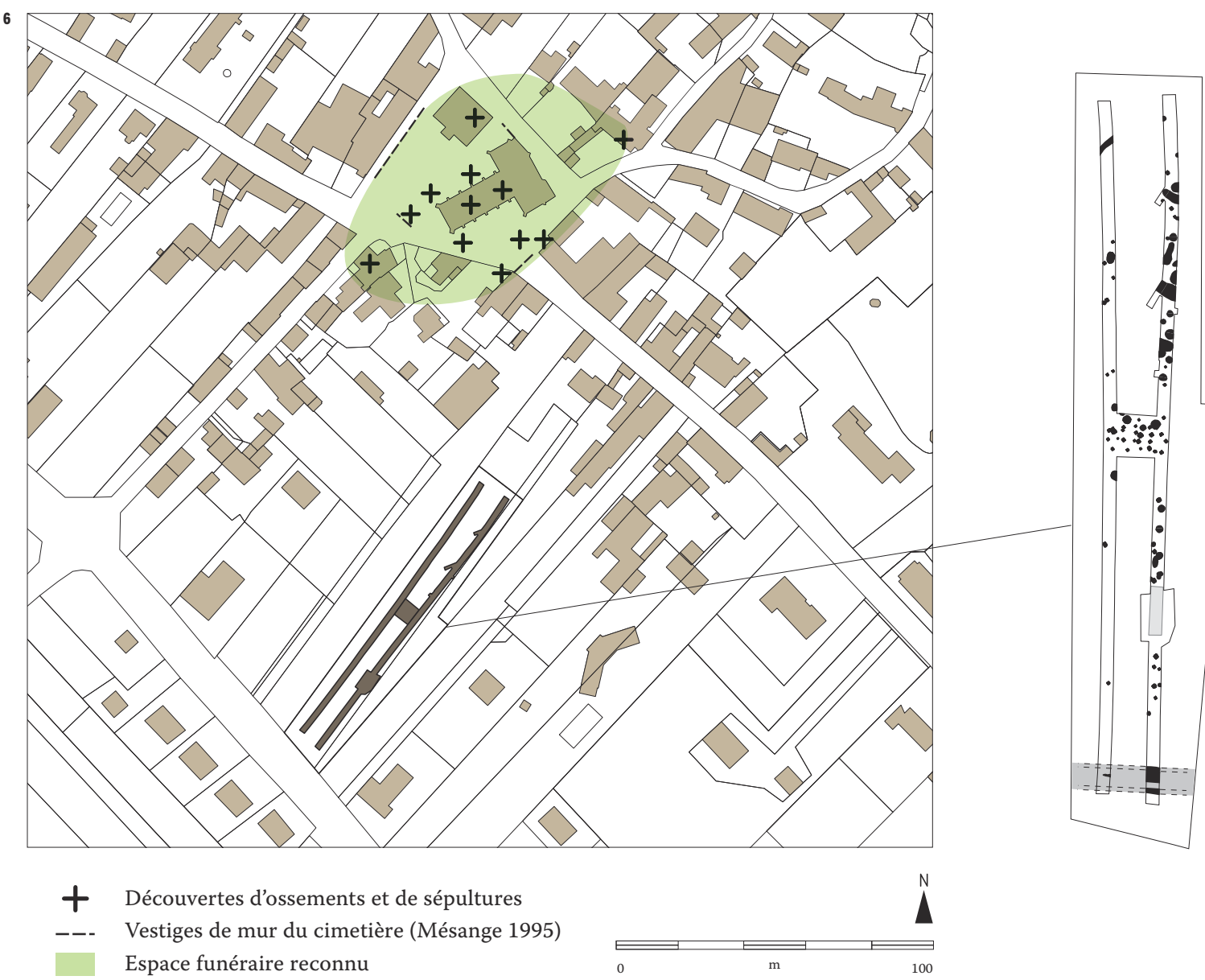

- Vestiges de mur du cimetière (Mésange 1995) Espace funéraire reconnu

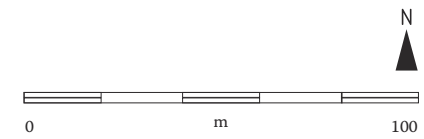


paroisses de Touraine (Zadora-Rio et al., 2008). Cette documentation est toutefois intégrée au cas par cas au sein des notices de l'Atlas archéologique de Touraine (Zadora-Rio, 2007-2014). Le projet collectif de recherche portant sur les agglomérations secondaires antiques de région Centre les a pris en compte dans les notices de sites (Bellet et al., 1999; Cribellier, à paraître) ou dans les travaux de synthèse (Jesset, 2012 ; Moreau, Zadora-Rio, 2012). Il s'agit toutefois ici d'un éclairage spécifique, celui $\mathrm{du}$ « devenir » des agglomérations de l'Antiquité aux périodes suivantes.

Malgré ses défauts intrinsèques, cette documentation est propice à la réalisation de synthèses monographiques. Bonnée et Esvres en sont des exemples révélateurs. Le site de Bonnée est connu depuis le XIX ${ }^{\mathrm{e}}$ siècle, mais jusqu'en 2001 sa documentation se résume à des découvertes fortuites, des relevés du XIX ${ }^{\mathrm{e}}$ siècle, des sources textuelles médiévales et une opération de sauvetage ponctuel. Sept diagnostics en une douzaine d'années renouvellent profondément les connaissances en confirmant l'hypothèse d'une agglomération antique et en dévoilant son évolution médiévale. L'accumulation de ces interventions a permis d'affiner la première synthèse établie dans le cadre d'une recherche collective (Cribellier, 1999, p. 199) et d'en proposer une cartographie précise (Joly et al., 2014). À Esvres, les données de l'archéologie préventive sont complétées par l'acquisition d'une documentation spécifique dans le cadre du programme de recherche. À chaque fois, une volonté partagée par les acteurs permet de dépasser l'opportunisme archéologique initial pour alimenter un état synthétique des connaissances et développer des réflexions spécifiques, seules à même d'aborder l'archéologie du village.

\section{Références bibliographiques}

HTSB, 1858, Historia translationis sancti Benedicti / ab Adrevaldo scripta, Paris, Société de l'histoire de France.

Jollois J.-B., 1836, Mémoires sur les Antiquités du Loiret, Paris.

BARTHÉLÉMY D., 1993, La Société dans le comté de Vendôme de l'an mil au XIV siècle, Paris, Fayard, 1119 p.

Bellet M.-E., Cribellier C., Ferdière A., 1999, Agglomérations secondaires antiques en région Centre, Tours, FERACF/Archea (coll. Suppl. à la Revue archéologique du centre de la France, 17), 224 p.

Carlier M., Ladureau P., 2008, Sougé-sur-Braye «Le Bourg» (Loir-et-Cher), Rapport final d'opération de fouille archéologique, Tours, Inrap/SRA Centre, $167 \mathrm{p}$.

Carré F., Hincker V., Mahé N., Peytremann É., Poignant S., Zadora-Rio É., 2013, «Histoire(s) de village(s). L'archéologie en contexte villageois, un enjeu pour la compréhension de la dynamique des habitats médiévaux», in MAHÉ-HourLIER N. Poignant S. (DIR.), Archéologie du village, archéologie dans le village dans le nord de la France ( $V^{e}$-XIII ${ }^{e}$ siècles), Actes de la table ronde de SaintGermain-en-Laye, 22-24 novembre 2007, SaintGermain-en-Laye, Afam (coll. Mémoires publiés par l'Association française d'archéologie mérovingienne, XXIX), p. 237-248.

Chimier J.-PH., 2012, Esvres, (Indre-et-Loire, région Centre), rue de la Passerelle, Rapport de sauvetage urgent et rapport de diagnostic, Tours, Inrap/SRA Centre, $58 \mathrm{p}$.

Chimier J.-Ph., Dubois J., Foulllet N., Pouyet T., 2014, «Esvres-sur-Indre, de la Protohistoire récente au début du Moyen Âge », in ZADORA-Rio E. (DIR.), Atlas archéologique de Touraine, [en ligne] $<$ http://a2t.univ-tours.fr/notice.php?id=65 >.

Chimier J.-PH., Fouillet N., 2012, Evena: évaluation archéologique d'une agglomération d'origine protohistorique, UMR 7324 Citeres, LAT, [en ligne] $<$ http://citeres.univ-tours.fr/spip.php?article1765 >
Chimier J.-Ph., Fouillet N., 2012, «Evena. Présentation du programme de recherche archéologique sur le village d'Esvres et son territoire communal et premiers résultats (2011-2012) ", Bulletin de la Société archéologique de Touraine, 58 , p. 101-108.

Chimier J.-Ph., RiQuier S., 2009, «L'organisation des espaces funéraires d'Esvres-sur-Indre (Indre-etLoire). État de la question sur les hypothèses de topographie funéraire et sur l'organisation territoriale des occupations protohistoriques et antiques ", Revue archéologique de Picardie, 29, Les gestuelles funéraires au second âge du Fer, p. 85-95.

Cribellier C., à paraître : Agglomérations secondaires antiques en région Centre, Orléans, FERACF/ Archea (coll. Suppl. à la Revue archéologique du centre de la France).

Cribellier C., 1999 : « Bonnée (Loiret) », in Bellet M.-E., Cribellier C., Ferdière A., Krausz S. (DIR.), Agglomérations secondaires antiques en région Centre, Tours, FERACF (coll. Suppl. à la Revue archéologique du centre de la France, 17) p. 195-197.

De Filippo R., 2012 : Bonnée (Loiret), $50 a$ route des Bordes (45.039.020AH), Arr-Presc-Diag 2012/o220, Rapport de diagnostic, Tours, Inrap, 33 p.

Fournier L., 2012 : Sougé, Loir-et-Cher, Le Bourg, Rapport de diagnostic, Orléans, Inrap/SRA Centre, $52 \mathrm{p}$.

JESSET S., 2012 : «Évolutions des agglomérations antiques du Loiret au haut Moyen Âge », in Cribellier C., Ferdière A. (Dir.), Agglomérations secondaires antiques en région Centre, 2, Actes de la table ronde d'Orléans, Tours, FERACF (coll. Suppl. à la Revue archéologique du centre de la France, 42), p. 111-130.

Joly S., Aunay C., Barret M., Chambon M.-P., LiARD M., Lusson D., 2014: Rue Creuse à Bonnée, (45.039.022.AH), un nouveau quartier de l'agglomération antique, Arr-Presc-Diag 13/o755, Rapport final d'opération de diagnostic archéologique, Tours, Inrap, $92 \mathrm{p}$

Joly S., Boulllon J., 2014 : 4 bis avenue du Petit Thouars à Villiers-sur-Loir (41.294.006.OP), Les prémices VIII ${ }^{e}-I X^{e}$ s. du village médiéval, Arr-PrescDiag 14/oo39, Rapport final d'opération de diagnostic archéologique, Tours, Inrap, $62 \mathrm{p}$
Joly S, Detante M, Fontaine A, Liard M, Mercey F., Millet S., 2008 : « Terres à l'est du Bourg» à Bonnée, Une occupation rurale du Bronze final IIIb et une occupation du début du haut Moyen Âge en bordure de l'agglomération antique, (45.039.018.AH), Arr-Presc-Diag 07/519, Rapport final d'opération de diagnostic archéologique, Tours, Inrap, 94 p.

LAULIAC E., 2013 : Église Saint-Médard, Esvres-surIndre (Indre-et-Loire), Mémoire de master 2, Université François-Rabelais, Tours.

Mahé-Hourlier N., Poignant S. (DIR.), 2013 Archéologie du village, archéologie dans le village dans le nord de la France (Ve-XIII ${ }^{e}$ siècles), Actes de la table ronde, 22-24 novembre 2007, SaintGermain-en-Laye, Saint-Germain-en-Laye, Musée d'archéologie nationale (coll. Mémoires publiés par l'Association française d'archéologie mérovingienne, XXIX), $249 \mathrm{p}$.

MÉSANGE H., 1995 : Villiers-sur-Loir, un village au fil des siècles, Vendôme, éd. du Cherche-Lune, $318 \mathrm{p}$.

Moreau A., Zadora-Rio E., 2012 : « Les trajectoires des agglomérations antiques de Touraine au Moyen Âge », in Cribellier C., Ferdière A. (DIR.), Agglomérations secondaires antiques en région Centre, 2, Actes de la table ronde d'Orléans, Tours, FERACF (coll. Suppl. à la Revue archéologique du centre de la France, 42), p. 131-144.

PAPIN P., 2013 : Joué-lès-Tours (37 22). Places VictorHugo et François-Mitterrand. Origine et évolution d'un centre paroissial rural (fin VII $-X I X^{e}$ s.), Rapport final d'opération de fouille archéologique, Tours, SRA Indre-et-Loire, 254 p.

Saint-Venant R. (de), 1917 : Dictionnaire topographique, historique, biographique, généalogique et héraldique du vendômois et de l'arrondissement de Vendôme, Blois, Migault.

THIÉRY G., 2006 : Sougé, « Le Bourg » (Loir-et-Cher), 41 250007 A.H., Rapport de diagnostic, Tours, Inrap/ SRA Centre, $32 \mathrm{p}$.

ZADORA-RIO E., 2010 : « Les ressorts administratifs du haut Moyen Âge : conditae et vicariae (VIII-X $X^{e}$ s.) », in ZADORA-RIo E. (DIR.), 2007-2014, Atlas archéologique de Touraine, Tours, ZadoraRio, [en ligne] < http://a2t.univ - tours.fr/notice. php?id=17\&menu $=$ Texte $>$.

Zadora-RIo E. (DIR.), 2008 : Des paroisses aux communes d'Indre-et-Loire. La formation des territoires, Tours, FERACF (coll. Suppl. à la Revue archéologique de la France, 34), 303 p.

ZADORA-RIO E. (DIR.), 2007-2014: Atlas archéologique de Touraine, Tours, Université de Tours, [en ligne] $<$ http://a2t.univ-tours.fr/index.php $>$. 